\title{
Structural Model of Eumelanin
}

\section{Citation}

Kaxiras, Efthimios, Argyrios Tsolakidis, George Zonios, and Sheng Meng. 2006.

"Structural Model of Eumelanin." Physical Review Letters 97 (21). https://doi.org/10.1103/ physrevlett.97.218102.

\section{Permanent link}

http://nrs.harvard.edu/urn-3:HUL.InstRepos:41384116

\section{Terms of Use}

This article was downloaded from Harvard University's DASH repository, and is made available under the terms and conditions applicable to Other Posted Material, as set forth at http:// nrs.harvard.edu/urn-3:HUL.InstRepos:dash.current.terms-of-use\#LAA

\section{Share Your Story}

The Harvard community has made this article openly available.

Please share how this access benefits you. Submit a story.

\section{Accessibility}




\title{
Structural Model of Eumelanin
}

\author{
Efthimios Kaxiras, ${ }^{1}$ Argyrios Tsolakidis, ${ }^{1, *}$ George Zonios, ${ }^{2}$ and Sheng Meng ${ }^{1}$ \\ ${ }^{1}$ Department of Physics and Division of Engineering and Applied Sciences, Harvard University, \\ Cambridge, Massachusetts 02138, USA \\ ${ }^{2}$ Department of Materials Science and Technology, University of Ioannina, Ioannina 45110, Greece
}

(Received 31 January 2006; published 21 November 2006)

\begin{abstract}
Melanin is a ubiquitous pigment in living organisms with multiple important functions, yet its structure is not well understood. We propose a structural model for eumelanin protomolecules, consisting of 4 or 5 of the basic molecular units (hydroquinone, indolequinone, and its tautomers), in arrangements that contain an inner porphyrin ring. We use time-dependent density functional theory to calculate the optical absorption spectrum of the structural model, which reproduces convincingly the main features of the experimental spectrum of eumelanin. Our model also reproduces accurately other important properties of eumelanin, including $\mathrm{x}$-ray scattering data, its ability to capture and release metal ions, and the characteristic size of the protomolecules.
\end{abstract}

DOI: $10.1103 /$ PhysRevLett.97.218102

PACS numbers: 87.15.Aa, 82.35.Pq, 87.15.Mi

Melanin is the most important chromophore in human skin and a ubiquitous pigment in animals, plants, and microorganisms, but its purpose and function [1,2], and its detailed structure and properties remain controversial $[3,4]$. In humans, melanin is synthesized in melanocytes and exists in two main forms, the brown-black eumelanin and the red-yellow pheomelanin; its synthesis begins with hydroxylation and oxidation of tyrosine leading to oligomers of indole units (in eumelanin) and benzothiazine derivatives (in pheomelanin) [5]. The widely accepted function of melanin is photoprotection from solar uv light [6,7], but a variety of other roles have also been proposed: skin coloration, camouflage, adornment, thermoregulation, vitamin D synthesis regulation, regulation of skin sensitivity to frostbite, antioxidant behavior, drug and metal-ion binding, and conversion of optical energy to electrical energy and heat $[1,2]$. Melanin has also been implicated in Parkinson's disease [8], age-related macular degeneration [9], and malignant melanoma [10], the most aggressive type of skin cancer. The lack of an established structural model makes the interpretation of melanin's properties and function problematic.

Native skin melanin is difficult to isolate and study. Alternative types of melanin have been employed as reliable models, including synthetic melanin, melanin from the ink sack of the cuttlefish Sepia officinalis, and melanin extracted from human and animal hair employing various chemical processing methods [11]. In eumelanin, the basic units are oligomers stacked and otherwise linked together to form a substance with the characteristics of amorphous semiconductors [12]. The consensus is that the molecular units comprising eumelanin are 5,6dihydroxyindolequinone or hydroquinone (DHI or HQ), its redox forms indolequinone (IQ) and tautomers quinone-methide (QI1) and quinone-imine (QI2), and 5,6-dihydroxyindole 2-carboxylic acid (DHICA). How these units are put together to form the oligomers (proto- molecules) of melanin has not yet been established, leaving a basic gap in our understanding of its structure.

We propose and study here a new model for the structure of the protomolecules of eumelanin. Our model provides a natural explanation of the basic characteristic properties of eumelanin. We support this proposal with extensive firstprinciples quantum mechanical calculations of the structural, electronic, and optical properties of the protomolecules, which are in excellent agreement with available experimental data.

We first identify the crucial characteristics of eumelanin, which a structural model should reproduce: (a) While x-ray studies from synthetic melanin and melanin from Sepia officinalis [13-16] cannot uniquely determine the structure, they do provide important constraints, of which some distinct features are the following: (i) the protomolecules are relatively small in size, which is interpreted to correspond to tetramers or pentamers of the HQ, IQ or QI monomers; (ii) the protomolecules appear to be stacked in planar graphiticlike arrangements. Existing atomic-scale models $[14,15,17,18]$ are not able to explain the finite size of the protomolecules, but rely on artificial constructs restricted to oligomers, by randomly linking the monomers. (b) The tautomers of IQ, QI1, and QI2 are present in chemical analysis of the melanin particles $[19,20]$. Thus, it is not unreasonable that they are also components in the melanin protomolecules. This is puzzling, however, because these particular monomers are shown by theoretical analysis $[21,22]$ to be higher in energy than the tautomeric indolequinone, and should therefore have small to negligible concentration (13\% for the methide form and $0.1 \%$ for the imine form, relative to IQ, at $300 \mathrm{~K}$ [22]). On the other hand, experiment also shows that these tautomers cannot be the sole ingredient of the protomolecules of melanin [19]. (c) Melanin has the ability to capture and release metal ions without any apparent change in morphology $[4,23]$; this has been interpreted as indicative of a 
structure which allows the efficient transport of metal ions without damage. A metal-ion binding capacity of one ion per 3-4 monomer units has been assigned by experiments [23]. (d) The most characteristic and challenging behavior of melanin is its very broad and smooth absorption spectrum in the uv and visible range, 700-200 nm (1.7$6.2 \mathrm{eV}$ ). This behavior is central to some of its biological functions, such as the blocking of uv radiation, and is puzzling because it lacks any of the sharp features typical of other key biomolecules in the uv range.

The basic idea behind our model for the melanin protomolecules is illustrated in Fig. 1: the four constituent molecules, IQ, HQ, QI1, and QI2, are linked by covalent bonds between the $\mathrm{C} 2$ and $\mathrm{C} 7$ atoms, exclusively. This creates an interior ring where all the $\mathrm{N}$ atoms reside, in an arrangement similar to porphyrin; that is, the $\mathrm{N}$ atoms are separated by three successive covalently bonded $\mathrm{C}$ atoms. In the tetramer, two adjacent monomers are positioned so that the major axis, which passes through $\mathrm{C} 2$ and bisects the bonds between $\mathrm{C} 8-\mathrm{C} 9$ in the pentagon and $\mathrm{C} 5$ $\mathrm{C} 6$ in the hexagon in one monomer, is perpendicular to the corresponding axis of the next monomer. We note that a tetramer is the smallest unit that can be formed in this manner. A trimer would put the two aforementioned axes on adjacent monomers at an angle of $60^{\circ}$, creating significant strain in the inner ring which would render this structure unstable. We have created pentamers following the same rule, and we find that, although less stable than the tetramers, they are not unstable due to strain (the two aforementioned axes of adjacent monomers are at a $108^{\circ}$ angle). On the other hand, hexamers are unstable, because the two axes of adjacent monomers are at an angle of $120^{\circ}$, which brings the $\mathrm{O} 6$ atom of one monomer close to the $\mathrm{H}$ $\mathrm{C} 3$ atom of the next, creating steric hindrance. Thus, the rule of creating rings in the manner described above natu-

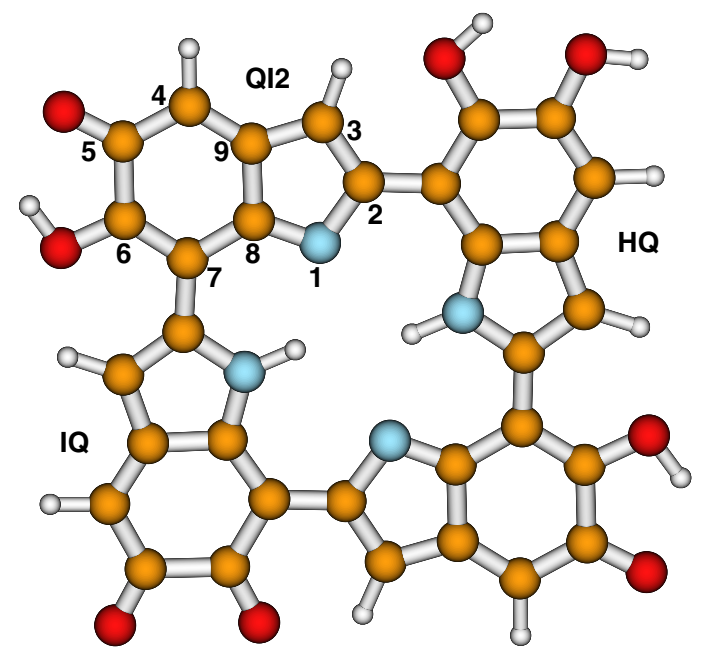

FIG. 1 (color online). A tetramer composed of IQ-QI2-HQ$\mathrm{QI} 2$ (gold $=\mathrm{C}$, red $=\mathrm{O}$, blue $=\mathrm{N}$, and white $=\mathrm{H}$ ). Atoms in the QI2 monomer are numbered in the standard notation. rally explains why the smallest protomolecule consists of four and the largest of five monomers, as suggested by the available experimental evidence [13-16]. This is already a significant improvement over any other attempt to create the oligomers out of the monomers in a natural manner, accounting for property (a) mentioned above. The formation pathway of such tetramers should also be favorable because the most stable binding sites to form a dimer are $\mathrm{C} 2$ and $\mathrm{C} 7$ [24]. In the following, we concentrate for simplicity on tetramer protomolecules.

There are 21 possible arrangements of four individual molecules in a tetramer if we restrict the QI form to one type (either methide, QI1, or imine, QI2). We have performed calculations to obtain the formation energy of tetramers starting with monomers in vacuum, and found that of the 21 possible tetramers, the eight that contain no QI have large negative formation energy (endothermic reaction), the six that contain one QI have small positive formation energy (weakly exothermic reaction), and the eight that have two or three QIs have large positive formation energy (strongly exothermic reaction). Finally, the one that contains exclusively four QIs has small positive formation energy. We employed two different and widely used methodologies to perform these calculations within first-principles density functional theory $[25,26]$, and the two sets of results, shown in Fig. 2, are in very good overall agreement.

These results give a remarkably clear explanation of experimental indications that QIs may play a role in the structure of eumelanin, even though they are energetically disfavored compared to the tautomeric indolequinone, as discussed in property (b) above: A tetramer without any QIs has $4 \mathrm{H}$ atoms attached to the $\mathrm{N}$ atoms in the inner ring, which produce strong steric repulsion. Even the presence

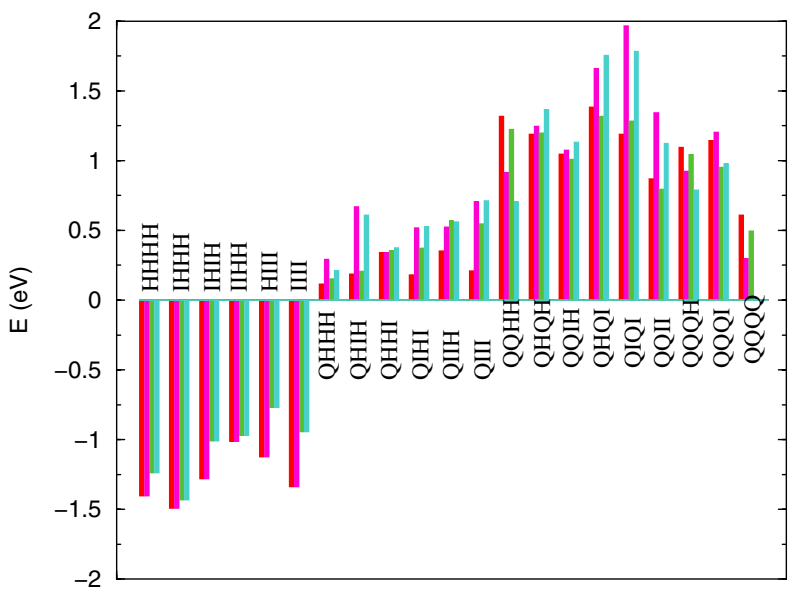

FIG. 2 (color online). Formation energies of the various tetramers labeled by the first letter of the constituent quinones, I for IQ, H for HQ, and Q for either QI1 or QI2. The bars correspond to results for the two different forms of QI, as obtained from the SIESTA code (first two, red-shaded bars) or from the VASP code (second two, green-shaded bars). 
of $3 \mathrm{H}$ atoms in the inner ring (only one QI unit in the tetramer) appears unfavorable. The presence of at least two or three QIs (only 1 or $2 \mathrm{H}$ atoms in the inner ring) is favorable. We emphasize that if QIs were not involved in the synthesis of tetramers it would not invalidate our model, which only relies on the assumption that the inner porphyrinlike ring contains at most $2 \mathrm{H}$ atoms bonded to $\mathrm{N}$ atoms. This could be the result of reactions that remove $\mathrm{H}$ atoms from $\mathrm{N}$ in the IQ or HQ units during or after formation of the ring. The existence of several structurally similar and energetically equivalent tetramers is consistent with the recently proposed chemical disorder model [27].

There are eight structures containing 1 or $2 \mathrm{H}$ atoms in the inner ring, among the total of 21 structures that we considered for each of the two types of QI. In the following, we concentrate on those structures; we refer to them as the dominant tetramers. It is also possible to create structures with mixed QI1 and QI2 units, which would further contribute to chemical disorder [27].

The calculated structure factor $S(q)$ and radial distribution function (RDF) based on our model are shown in Fig. 3. The RDF is derived through Fourier transform of $S(q)$ and contains an additional term $4 \pi r^{2} \rho_{s}$, with $\rho_{s}$ the average density, following the procedure of Ref. [14] with the same parameters. The $3 \mathrm{D}$ stacking of tetramers is
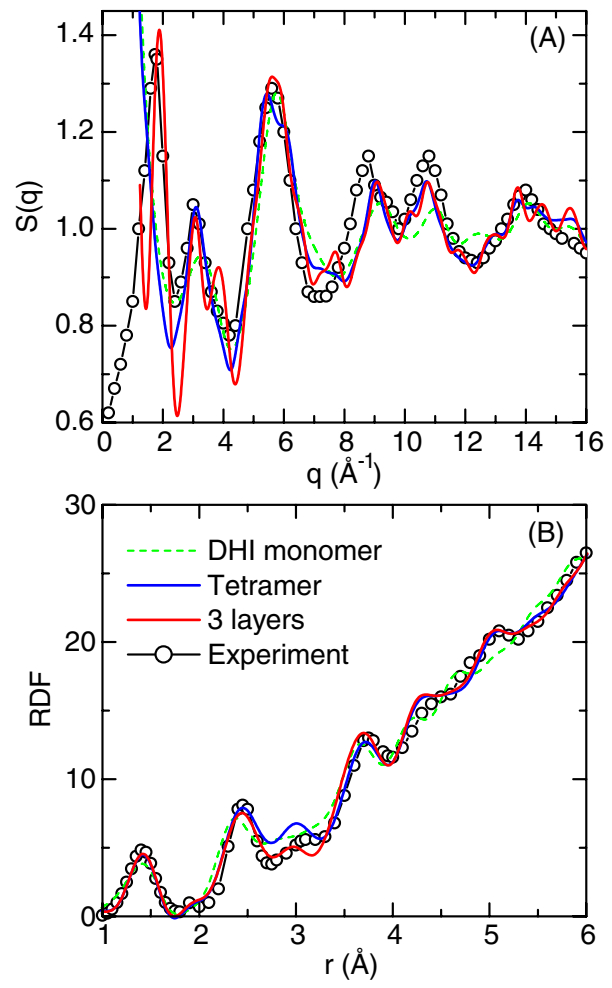

FIG. 3 (color online). Calculated structure factors $S(q)$ and radial distribution functions (RDF) of the DHI monomer, the tetramers, and the three-layer model. Experimental data are presented for comparison (from [14]). The tetramer curves are averaged over the dominant structures (see text). essential to reproduce the very first peak in the experimental $S(q)$. We therefore form 3D arrangements by stacking three layers of the planar tetramer units and vary the relative distances and orientation between the layers. In the optimal arrangement, the tetramers are shifted by $3.1 \AA$ in both lateral directions and rotated by $30^{\circ}$ between adjacent layers, which are separated by $3.3 \AA$. The RDF for this arrangement produces an excellent fit to the experimental data. In addition, the resulting density of $1.30 \mathrm{~g} / \mathrm{cm}^{3}$ agrees well with the experimental value for tyrosine melanin $\left(1.27 \mathrm{~g} / \mathrm{cm}^{3}\right)$ [14]. A significant success of the present model is the peak around $3.00 \AA$, which no other model reproduces adequately [14]. This peak is due to the presence of the inner ring in the tetramer and corresponds to distances between the $\mathrm{N}$ atoms and their third neighbors in the inner ring (the $\mathrm{C} 2$ or $\mathrm{C} 8$ atoms in the neighboring monomers). We emphasize that only a regular structure like the porphyrinlike inner ring can produce this feature in the RDF, which is lost when the monomers are linked in a more random fashion. In fact, existing structural models produce a second peak in the RDF which is broader than the measured peak [14], precisely because they lack the regularity of the inner ring structure, whereas the second peak produced by our model is as sharp as the first peak, as in experiment $[13,14]$. Nevertheless, it is plausible that other structural models could also reproduce experiments well.

Our model also accounts naturally for the capture and release of metal ions by melanin. The inner ring of the tetramer structure we propose is identical to that in porphyrin. Three of the eight dominant tetramers, QHQH, QHQI, and QIQI, which have the highest formation energy, contain exactly two nonadjacent $\mathrm{H}$ as porphyrin does. It is known that porphyrin can capture and release a variety of metal ions. Consequently, the tetramers should exhibit similar behavior. This provides a natural explanation of the property (c). Indeed, the reported binding capacity of one ion per 3-4 monomer units [23] is in agreement with our model.

Finally, a crucial test of the atomic-scale structure of melanin is whether it can reproduce the experimentally observed optical absorption spectrum. Accurate calculations of optical absorption spectra are computationally very demanding. We have performed such calculations based on time-dependent density functional theory, which reproduces well the experimental spectra of various biological molecules [28]. The results are shown in Fig. 4. The individual tetramers have of course sharp features, as do all biological molecules with specific structure. However, a structure formed by putting together a random collection of the dominant tetramers, stacked in units of three or four high in a manner analogous to graphite, is likely to show no sharp features. An average of the spectra of the 16 dominant tetramers we have studied produces a largely featureless spectrum, except for two weak and broad shoulders at 


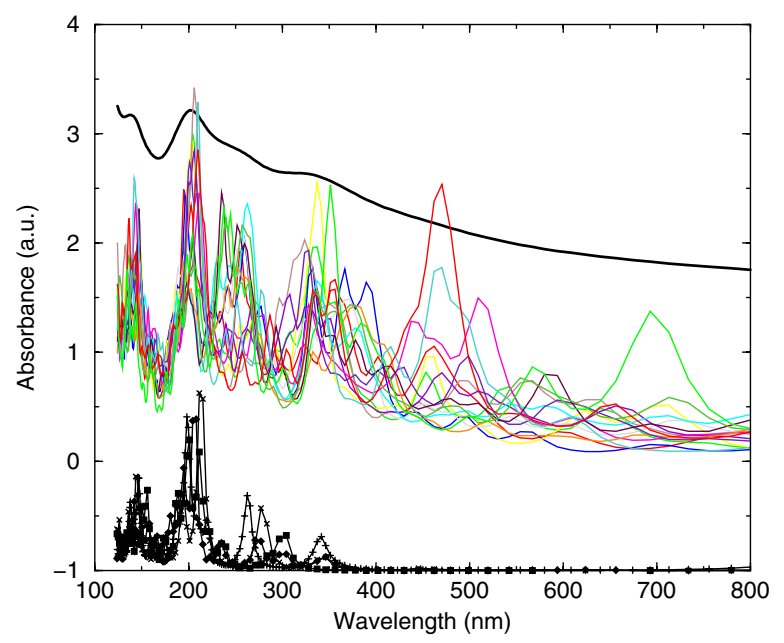

FIG. 4 (color online). Absorption spectrum of the superposition of dominant tetramers (thick black line, shifted up by 1.5 units for clarity). Individual tetramer contributions are given by the thin colored lines and monomer spectra are also shown (shifted down by 1 unit for clarity).

around $350 \mathrm{~nm}$ and around $240 \mathrm{~nm}$. They are also observed in some experimental studies [27], mainly for melanin structures with incomplete oxidization. It is quite remarkable that individual tetramers have sharp features, but these are eliminated when the average is taken, in agreement with the chemical disorder model [27]. Thus, our model produces an optical absorption spectrum entirely consistent with experiment, as discussed in property (d) of melanin. The spectra of the four individual monomers are also shown in Fig. 4. By comparison, the absorption by monomers has completely died out at about $350 \mathrm{~nm}$ and all absorption beyond this range is due to delocalized electronic states introduced by the tetramer structure. The range below $200 \mathrm{~nm}$, where all monomers have strong absorption, is typically cutoff in experimental measurements.

The slope of the calculated optical absorption curve indicates that the structure proposed here would correspond to very dark (black) color, consistent with experimental reports for DHI-rich eumelanin [29]. We expect the presence of pentamers and related variations of the simple tetramer structure to produce further broadening of the spectrum, in accordance with the chemical disorder model [27]. We have also constructed planar hydrogen-bonded arrangements of the tetramers, which indicates that large two-dimensional structures can be formed easily. Such arrangements may also contribute to broadening of the absorption spectrum. Moreover, we have not directly addressed the issue of melanin derived from DHICA, which generally has lighter color [29] and is likely to have a different structure. The model proposed here provides a basis for a theory of the properties and biological function of melanin.

We thank Paul Maragakis for useful discussions.
*Current address: Shell International Exploration and Production BV, Kesslerpark 1, 2288 GS Rijswijk (ZH), The Netherlands.

[1] W. L. Morison, Arch. Dermatol. 121, 1160 (1985).

[2] H.Z. Hill, BioEssays 14, 49 (1992).

[3] W. L. Cheun, K. Kim, and J. Shao, Pigment Cell Res. 17, 422 (2004).

[4] J. D. Simon and S. Ito, Pigment Cell Res. 17, 423 (2004).

[5] S. Ito, Pigment Cell Res. 16, 230 (2003).

[6] N. Kollias, R. M. Sayre, L. Zeise, and M. R. Chedekel, J. Photochem. Photobiol., B 9, 135 (1991).

[7] J.-P. Ortonne, British J. Dermatol. 146, 7 (2002).

[8] F. A. Zucca et al., Pigment Cell Res. 17, 610 (2004).

[9] T. T. J. M. Berendschot et al., Invest. Ophthalmol. Visual Sci. 43, 1928 (2002).

[10] S. Pavel et al., Eur. J. Cancer 40, 1423 (2004).

[11] S. Ito and K. Wakamatsu, Pigment Cell Res. 16, 523 (2003).

[12] J. McGinness, P. Corry, and P. Proctor, Science 183, 853 (1974).

[13] J. Cheng, S. C. Moss, and M. Eisner, Pigment Cell Res. 7, 255 (1994).

[14] J. Cheng, S. C. Moss, M. Eisner, and P. Zschack, Pigment Cell Res. 7, 263 (1994).

[15] G. W. Zajac et al., Biochim. Biophys. Acta 1199, 271 (1994).

[16] G. W. Zajac, J. M. Gallas, and A. E. Alvarado-Swaisgood, J. Vac. Sci. Technol. B 12, 1512 (1994).

[17] K. B. Stark et al., J. Phys. Chem. B 107, 11558 (2003).

[18] K. B. Stark et al., J. Phys. Chem. B 109, 1970 (2005).

[19] Ch. Lambert et al., Biochim. Biophys. Acta 993, 12 (1989).

[20] A. T. Al-Kazwini et al., J. Phys. Chem. 94, 6666 (1990).

[21] Y. V. Il'ichev and J. D. Simon, J. Phys. Chem. 107, 7162 (2003).

[22] B. J. Powell et al., J. Chem. Phys. 120, 8608 (2004).

[23] Y. Liu et al., Pigment Cell Res. 17, 262 (2004).

[24] K. B. Stark et al., J. Phys. Chem. B 107, 3061 (2003).

[25] D. Sánchez-Portal, P. Ordejón, E. Artacho, and J. M. Soler, Int. J. Quantum Chem. 65, 453 (1997). We used TrouillerMartins pseudopotentials, the local density approximation exchange-correlation functional of the Ceperley-Alder form, and a basis of double zeta polarized orbitals (13 atomic orbitals for $\mathrm{C}, \mathrm{N}$, and $\mathrm{O} ; 5$ orbitals for $\mathrm{H}$ ).

[26] G. Kresse and J. Furthmuller, Phys. Rev. B 54, 11169 (1996). We used a $520 \mathrm{eV}$ plane wave cutoff, projected augmented wave pseudopotentials, with the same exchange-correlation functional as in the SIESTA calculations, a converged unit cell of dimensions $21 \times 21 \times 14 \AA$ (the molecules lie on the $x y$ plane) and the $\Gamma$ point (center) of the Brillouin zone for reciprocal space sampling.

[27] M. L. Tran, B. J. Powell, and P. Meredith, Biophys. J. 90, 743 (2006).

[28] For details of the methodology, see A. Tsolakidis and E. Kaxiras, J. Phys. Chem. A 109, 2373 (2005). We used 12212 steps in time to propagate the wave functions with time step $21.28 \times 10^{-3} \mathrm{fs}$, which gives $0.05 \mathrm{eV}$ energy resolution and perturbing external field $0.1 \mathrm{~V} / \AA$.

[29] S. J. Orlow, M. P. Osber, and J. M. Pawelek, Pigment Cell Res. 5, 113 (1992). 